\title{
Técnicas da Pesquisa Operacional Aplicadas na Otimização do Fluxo de Pacientes do Sistema Único de Saúde do Estado do Paraná
}

C.T. SCARPIN, G.J.C. DIAS, UFPR, Programa de Pós-Graduação em Métodos Numéricos em Engenharia, Cx.P. 19081, 81531-990 Curitiba, PR, Brasil.

M.T. ARNS STEINER, V.E. WILHELM, UFPR, Departamento de Matemática e Programa de Programa de Pós-Graduação em Métodos Numéricos em Engenharia, Cx.P. 19081, 81531-990 Curitiba, PR, Brasil.

P.J. STEINER NETO, UFPR, Departamento de Administração e Programa de Pós-Graduação em Administração, Cx.P. 19081, 81531-990 Curitiba, PR, Brasil.

\begin{abstract}
Resumo. O presente trabalho apresenta uma solução otimizada para o fluxo de pacientes que procuram atendimento junto ao Sistema Único de Saúde (SUS) do Estado do Paraná, Brasil, cujas cidades de origem não possuem o procedimento médico necessário. A solução deve levar em consideração além do procedimento requerido, também, a hierarquia em relação à regionalização que, em ordem crescente é: cidade, micro-região, região/regional e macro-região, a partir do local de origem do usuário até o seu destino final, assim como a capacidade de atendimento do local de destino. A problemática consiste em obter a definição do fluxo ótimo dos usuários do SUS a partir do local de origem até os centros de atendimento mais próximos, obedecendo as restrições anteriormente mencionadas, de forma a atendê-los o mais rapidamente possível. Este objetivo pode ser alcançado através de um sistema computacional, contendo um algoritmo matemático, que organize e controle adequadamente os dados da Secretaria de Saúde do Estado do Paraná (SESA); como conseqüência, obter-se-á a otimização dos recursos do SUS destinados aos municípios. A ferramenta desenvolvida, baseada em técnicas da Pesquisa Operacional, é de fácil acesso e de grande eficiência, apresentando resultados significativamente superiores aos atuais.
\end{abstract}

Palavras-chave. Problema de $p$-medianas, fluxo de pacientes, rota mínima.

\section{Introdução}

O Sistema Único de Saúde do Estado do Paraná (SUS), através da Secretaria de Saúde (SESA), busca uma solução para o problema de fluxo de pacientes que não encontram o procedimento médico necessário em sua cidade/município de origem e que necessitam ser encaminhados para outra cidade do estado para atendimento. 
Atualmente, este fluxo não possui uma ordem pré-definida, nem tampouco uma orientação das próprias secretarias municipais para onde devem se encaminhar. Como conseqüência, os grandes centros de atendimentos, especialmente a cidade de Curitiba, PR, Brasil, ficam com super lotações de pessoas em seus hospitais, o que dificulta e prejudica o atendimento e a sua organização, gerando prejuízos na qualidade dos serviços prestados à população.

O sistema de saúde no Brasil possui três níveis de resolutividade para os procedimentos médicos ofertados pelo estado: baixa, média e alta complexidade [7]. Assim, procedimentos de menores níveis de resolutividade, poderiam ficar ao encargo de cidades menores e os procedimentos de níveis de mais alta complexidade, seriam destinados às cidades maiores.

Um projeto de regionalização do estado está sendo estudado pela SESA, visando distribuir os níveis de resolutividade de procedimentos em grupos de cidades da seguinte forma: um certo número de cidades formaria um grupo maior, chamado de micro-região, onde a cidade-sede ficaria responsável pelos procedimentos de baixa complexidade; um certo número de micro-regiões formaria um grupo maior, chamado de região/regional, no qual a cidade-sede ficaria responsável pelos atendimentos de média complexidade; e a junção de regionais, que formaria um grupo maior de cidades chamadas de macro-regiões, nas quais a cidade-sede seria responsável pelos procedimentos médicos ofertados pelo estado de alta complexidade.

Diante deste contexto, este trabalho apresenta um algoritmo lógico/matemático [1] que considera a estrutura acima sugerida e define a designação/fluxo otimizada dos pacientes, assim como o controle dos procedimentos realizados em cada cidade até a divisão hierárquica do estado, de forma a minimizar a distância a ser percorrida pelos usuários do SUS.

O presente trabalho está organizado da seguinte forma: na seção 2 está a descrição do problema e o levantamento de dados, dando um enfoque geral do problema para uma melhor compreensão da complexidade de implementação e obtenção da solução. Na seção 3 é apresentada uma proposta do algoritmo para a otimização do fluxo dos pacientes, baseado no Algoritmo de Floyd ([3], [6]) de forma a se obter a menor distância entre as cidades e, consequentemente, a designação otimizada dos pacientes. Como conseqüência, obter-se-á a otimização na deliberação dos recursos destinados pelo SUS aos diversos municípios. Algumas simulações da utilização do

algoritmo são apresentadas na seção 4. Na seção 5, apresentam-se as conclusões e uma breve análise de comparação do fluxo otimizado com o fluxo atual de usuários.

\section{Descrição do Problema e Levantamento de Da- dos}

Quando um paciente recorre a um posto médico de sua cidade, a procura de um procedimento médico, o mesmo espera obter o atendimento o mais rapidamente possível, pois trata-se de um problema de saúde. Fica explícita a urgência de atendimento. No caso da cidade oferecer o procedimento médico requerido, a solução fica fácil, a não ser pela existência de fila de espera, o que ocorre muitas vezes. No entanto, no caso da cidade não possuir o procedimento requerido (por falta de um 
médico especialista e/ou de aparelhagem para um determinado exame específico), os funcionários das secretarias municipais de saúde deveriam encaminhar esse paciente para uma outra cidade, que ofereça o referido procedimento e que tenha capacidade para tal. Esse encaminhamento deve ser o mais rápido possível, para que a enfermidade do paciente não se agrave ou, ainda, não haja o risco de contágio, se for o caso.

A SESA oferece hoje cerca de 6.000 procedimentos médicos, sendo em diferentes quantidades e tipos em cada cidade. Estes procedimentos são divididos em dois grandes grupos: ambulatoriais (aproximadamente 3.500) e hospitalares (aproximadamente 2.500). Em cada grande grupo, existem grupos e sub-grupos menores que são divisões relacionadas às características do atendimento. Para exemplificar, tem-se nos procedimentos ambulatoriais, um grupo denominado "as ações executadas por profissionais da enfermagem"e, neste grupo, por sua vez, estão contidos os sub-grupos: "imunização"e "outras ações realizadas por enfermeiro(a)".

Cada cidade do Estado do Paraná possui estrutura e verba provinda do SUS, diferenciadas de acordo com os procedimentos médicos ofertados, destinadas à saúde. Entretanto, cidades como Ouro Verde do Oeste, PR, que não possui uma quantidade mínima de procedimentos, nem mesmo as de níveis baixos de resolutividade, tornase totalmente dependente da sede de sua micro-região, a cidade de Toledo, PR. Por outro lado, a cidade de Curitiba, possui todos os procedimentos ofertados pela saúde pública. Estes exemplos mostram que o estado apresenta grande variabilidade na oferta de seus serviços. Um dos requisitos para uma cidade receber verba do SUS é assumir a responsabilidade de ser sede de uma das divisões hierárquicas do estado, ofertando as chamadas especialidades, que são grandes grupos de procedimentos destinados somente a uma área da medicina.

O estado possui, atualmente, 15 grupos de especialidades: Cardiologia, Cirurgia Geral, Cirurgia Vascular, Dermatologia, Endocrinologia, Gastroenterologia, Ginecologia, Neurologia, Oftalmologia, Ortopedia, Otorrinolaringologia, Pneumologia, Psiquiatria, Reumatologia e Urologia. Cada especialidade é um conjunto de procedimentos, diretamente relacionados à sua área e é, através delas, que se tem o parâmetro para verificar se a mesma possui capacidade para assumir os atendimentos de baixa, média ou alta complexidade.

Cada especialidade possui, em média, 80 procedimentos e, além disso, quantidades mínimas de procedimentos de determinado nível. Assim sendo, a cidade pode ser autorizada (ou não) a ser responsável pela oferta da(s) especialidade(s), e, por conseguinte, pelo respectivo recebimento da verba do SUS. Estas ações devem ser aprovadas em reunião da BIPARTITE do Conselho Estadual de Saúde, que homologada a decisão em ata.

A obtenção de dados junto a SESA não foi uma tarefa fácil, pois não existe um controle da mesma sobre cada cidade: quantidades e procedimentos realizados; especialidades e níveis de resolutividade existentes; verba recebida pelo SUS; dentre outros. Informações revelam que existem inúmeros casos de procedimentos iniciados em certas cidades que, depois de um certo tempo, por diversos motivos, deixaram de ser realizados. No entanto, como a verba já estava sendo destinada àquela cidade, não existe forma de redirecioná-la, por dois principais motivos: 1 . porque a verba destinada à saúde ainda não é suficiente para cobrir todos os custos operacionais 
do estado; e 2. essa verba é destinada às cidades através de um pacote único para a saúde, sendo que não é especificado para que e de que modo deve ser gasta. Ao final de cada mês, as cidades prestam contas à SESA, porém não há nenhum instrumento para averiguar a veracidade das informações, ou seja, se a verba foi destinada de acordo com as informações.

Conforme comentado anteriormente, as cidades enviam informações à SESA sobre os procedimentos realizados. Vale salientar, que esse levantamento não é feito diretamente, mas pelas divisões hierárquicas do estado, isto é, cada cidade-sede de micro-região é responsável para a obtenção dos dados das cidades de sua microregião que, por sua vez, encaminha os dados para a cidade-sede da sua regional e, assim, que encaminha os dados à cidade-sede de sua macro-região e, finalmente, à SESA. Estas são as informações que foram consideradas no presente trabalho; as mesmas foram "tratadas"para tê-las de uma forma organizada, realizando-se um levantamento dos procedimentos médicos que cada cidade oferece.

Além das informações obtidas juntamente à SESA, obteve-se através do Departamento Nacional de Infra-estrutura do Transporte (DNIT), órgão federal, as distâncias entre as cidades do estado do Paraná para que pudessem ser utilizadas no cálculo da menor distância a ser percorrida pelo paciente para ser atendido.

Desta forma, tem-se todas as informações necessárias para o desenvolvimento do trabalho: o estado do Paraná é dividido em 6 macro-regiões, 22 regionais, 83 micro-regiões, e, aproximadamente, 6.000 procedimentos distribuídos entre as 399 cidades de formas diversas. Os procedimentos básicos, conforme já mencionado, são oferecidos por quase a totalidade de cidades existentes no estado, totalizando, deste modo, aproximadamente 350.000 registros de cidade-procedimento, existindo ainda, um controle de quais cidades realizam as especialidades e em que nível de resolutividade. Gerou-se, ainda, uma matriz de distâncias entre todas as cidades, da ordem $399 \times 399$.

Todas essas informações totalizam quase 600.000 registros que foram relacionados computacionalmente e fornecem, em um pequeno intervalo de tempo, cerca de 2 segundos, a solução otimizada para o paciente, ou seja, para qual cidade (que possui o procedimento de que ele necessita e capacidade para tal) ele deverá ser encaminhado de forma que o trajeto seja mínimo, para que o atendimento possa ocorrer o mais rapidamente e satisfatoriamente possível.

\section{Descrição do Algoritmo}

Como o número de registros para o controle é grande (cerca de 600.000) e os relacionamentos entre os mesmos são muitos, ao se apresentar os dados de entrada ao algoritmo ([2], [4]) com a cidade de origem e o procedimento requerido, fazse um filtro nos mesmos para que se trabalhe apenas com os registros necessários à designação do paciente. Assim sendo, armazenou-se as cidades que possuem o procedimento requerido e os dados das referidas cidades (localização; micro, regional e macro a que pertence) que o possuem, para que a busca seja otimizada, minimizando-se o número de registros.

É necessário obedecer a hierarquia para procurar o procedimento requerido, 
portanto, primeiramente verifica-se se a cidade de origem possui o procedimento. Se a resposta for afirmativa, observa-se se a sua capacidade de atendimento ainda possui disponibilidade no período (semana, mês ou outro, conforme definido pela SESA) e, se a resposta for novamente afirmativa, deve-se encaminhar o paciente para o local pré-definido pela Secretaria Municipal de Saúde da referida cidade para atendimento, com a autorização documentada em mãos, para realização do procedimento. Se ocorrer da cidade de origem não possuir o procedimento e/ou não houver capacidade para atender o paciente dentro do período e/ou, ainda, for um atendimento de emergência, é necessária a verificação de disponibilidade de atendimento/capacidade nas cidades pertencentes a mesma micro-região da cidade de origem do paciente, identificando, dentre elas, a cidade mais próxima da cidade de origem, colocando-a como $1^{\mathrm{a}}$ opção; a segunda mais próxima, como $2^{\mathrm{a}}$ opção, e assim sucessivamente. Caso a micro-região não possua o atendimento/capacidade necessários, relaciona-se as cidades pertencentes a mesma regional da cidade de origem. Caso ocorra a negativa novamente, procura-se dentre as cidades da macroregião e, se necessário, finalmente, para as cidades que não tenham vínculo com a cidade de origem, mas que tenham o procedimento, visando encontrar uma cidade que atenda as necessidades do atendimento solicitado.

Verificando a capacidade de atendimento da $1^{\mathrm{a}}$ opção e ocorrendo a autorização (pois há capacidade), encaminha-se o paciente para esta cidade; se não houver capacidade, analisa-se a $2^{\mathrm{a}}$ opção e assim por diante, até encontrar uma cidade que possa atender o paciente e que o encaminhamento possa ser autorizado. A cada resposta negativa das cidades pesquisadas como possíveis opções, faz-se a inserção das mesmas no relatório final, com o nome da pessoa responsável pela não autorização, para que se possa, deste modo, conhecer as limitações dos procedimentos e das capacidades de cada cidade.

Este procedimento possibilitará a emissão de relatório mensal que as cidades podem enviar à SESA para a checagem de dados e a identificação de discordância de dados. O procedimento sugerido faz com que seja obrigatória a procura de atendimento nas cidades mais próximas da cidade de origem, respeitando a hierarquia que existe entre as cidades a qual foi decidida em reuniões da BIPARTITE. As distâncias calculadas $(399 \times 399)$ são baseadas no Algoritmo de Floyd, que determina a menor distância entre quaisquer dois pontos (no caso, duas cidades) de um grafo $G(X, A)$, onde $X$ é o conjunto das 399 cidades e $A$ é o conjunto de arestas que as une.

\section{Composição do Algoritmo}

O algoritmo para a otimização do fluxo dos pacientes do SUS, ou seja, para a designação otimizada dos mesmos às cidades, consta das seguintes etapas:

- Dados de entrada: dados do paciente, cidade de origem e procedimento requerido;

- Faz-se a filtragem das cidades que possuem o procedimento requerido e de seus dados; 
- Se a cidade de origem consta do filtro, deve-se colocá-la como $1^{\mathrm{a}}$ opção;

- Caso a cidade de origem não conste do filtro, faz-se a verificação das cidades do filtro que pertençam a sua micro-região e que possuam o procedimento requerido. Coloca-se as mesmas em uma lista, em ordem crescente de distância, e, por conseguinte, de opção;

- Nas cidades do filtro que pertencem a sua regional e a sua macro-região, faz-se a mesma pesquisa em relação as distâncias e prossegue-se com a relação de opções;

- Caso a lista continue vazia, procede-se analogamente nas demais cidades do filtro;

- Em cada uma das checagens anteriores com relação as opções, verifica-se a capacidade de atendimento:

- se possuir, deve-se autorizar e encaminhar o paciente;

- se não possuir, deve-se inserir a cidade no relatório final e prosseguir com a lista de opções;

- se não houver opção alguma (lista vazia), ou seja, nenhuma cidade pode atender o paciente então:

* encaminhar o paciente para o próximo período, ou,

* encaminhar o paciente para o órgão estadual competente para prosseguir o atendimento.

\section{Simulações}

A seguir são apresentadas três simulações (exemplos) para melhor esclarecer o algoritmo apresentado.

Simulação 1: Para onde se deve encaminhar um paciente que necessita do procedimento "consulta em cardiologia", cód.0701205, que mora na cidade de Paranavaí, PR? O sistema computacional, contendo o algoritmo apresentado, filtrará (listará) todas as cidades que possuem o procedimento "consulta em cardiologia", cód. 0701205.

\section{Processo de Busca:}

- Em Paranavaí existe tal procedimento ("consulta em cardiologia", cód.0701205)? Sim (procura na tabela: linha do procedimento, coluna de Paranavaí);

- Há capacidade de atendimento?

Sim, encaminha para o local capacitado, mais próximo do local onde se encontra o paciente, o qual recebe um documento da secretaria municipal autorizando o atendimento. 
Simulação 2: Para onde se deve encaminhar um paciente que necessita do procedimento "cardioplastia", cód.33001049, que mora na cidade de Inácio Martins, PR?

Novamente, o sistema computacional, contendo o algoritmo apresentado, filtrará todas as cidades que possuem o procedimento "cardioplastia", cód.33001049.

\section{Processo de Busca:}

- A cidade de Inácio Martins possui tal procedimento? (busca no filtro) Não;

- A micro-região a qual pertence a cidade de Inácio Martins (este município pertence a duas micro-regiões: 1) micro-região de Irati que é composta das seguintes cidades: Irati, Mallet, Rio Azul, e outras e 2) micro-região de Rebouças: Rebouças, Imbituva, Guamiranga, e outras), possui alguma cidade com tal procedimento?

Não;

- A regional de Inácio Martins (regional 4: Irati, Imbituva, Fernades Pinheiro, e outras) possui?

Não;

- A macro-região dos Campos Gerais (Ponta Grossa: Irati, Inácio Martins, e outras) possui?

Sim; as cidades de Ponta Grossa, Telêmaco Borba e Arapoti possuem o procedimento solicitado;

- Destas três cidade qual é a mais próxima?

É a cidade de Ponta Grossa;

- A cidade de Ponta Grossa possui capacidade?

Não;

- Das duas cidades restantes (Telêmaco Borba e Arapoti) qual é a mais próxima? É a cidade de Telêmaco Borba;

- A cidade de Telêmaco Borba possui capacidade?

Sim; encaminhar o paciente com a respectiva documentação.

Simulação 3 (de forma mais resumida): Procedimento: "consulta em genética clínica", cód.0701216; cidade de origem: Faxinal, PR. O sistema computacional filtrará todas as cidades que possuem o procedimento "consulta em genética clínica", cód.0701216, que são: Curitiba, Francisco Beltrão, Pranchita, Umuarama, Marialva (filtro).

\section{Processo de Busca:}

- Início da busca: a cidade de Faxinal possui tal procedimento? Não; 
- Na micro-região da cidade de Faxinal existe alguma cidade que possui tal procedimento (micro de Apucarana: Apucarana, Cambira, Faxinal, Marumbi, Rio Bom, e outras)?

Não;

- A regional onde está localizado Faxinal (regional 16: Apucarana, Cambira, Faxinal, Marumbi, Rio Bom, Arapongas, Sabaudia, e outras) possui alguma cidade que possui tal procedimento?

Não;

- A macro-região de Faxinal (macro-região de Londrina: Apucarana, Londrina, Rolândia, Bandeirantes, Ibaiti, Ivaiporã, e outras) possui?

Não.

- Qual a cidade mais próxima?

- Verifica-se entre as cidades do filtro qual a cidade mais próxima da cidade de Faxinal, através do Algoritmo de otimização de Floyd ou por inspeção, que possui tal procedimento em ordem crescente de distância: Marialva, Umuarama, Curitiba, Francisco Beltrão, Pranchita.

- Se Marialva tiver capacidade de atendimento designa-se o paciente; caso contrário, verifica-se se a cidade seguinte, Umuarama, se possui capacidade, e assim por diante.

\section{Conclusões}

O sistema computacional contendo algoritmo lógico/matemático [8] apresentado neste trabalho, explora toda a capacidade de atendimento público na área de saúde no Estado do Paraná de forma hierárquica, verificando se existem outras possibilidades de atendimento médico que não os grandes centros, dentre os quais, principalmente, a cidade de Curitiba. Deste modo, o fluxo de pacientes que percorre o estado, a procura de atendimento com relação a certo procedimento médico irá, certamente, melhorar significativamente, pois na maior parte dos casos, o procedimento requerido não é exclusividade de uma cidade grande.

Assim, nos casos de procedimentos de alta complexidade, em que só a capital possui o atendimento, os hospitais não estarão com suas lotações esgotadas, ocupadas por procedimentos mais simples e poderão, em um prazo menor de tempo em relação ao atual, atender o paciente.

O algoritmo implementado traz melhoramentos, também, em outros aspectos do sistema de saúde como, por exemplo, as viagens de ambulâncias, que serão em menor número e percorrerão menores distâncias, pois em um grande número de casos, é o estado que arca com a despesa do serviço de translado do paciente. Ter-se-á, ainda, melhoramentos no controle e organização da verba pública destinada às cidades, devido ao maior número de informações e com maior nível de detalhamento que a SESA poderá obter, favorecendo, desta forma, o controle dos gastos e promovendo uma distribuição de verbas de forma coerente com a necessidade real de cada uma das cidades. 
Este controle possibilitará a alocação de recursos financeiros em cidades que ainda não possuem os procedimentos que lhes são necessários, assim como, em suas sedes, da micro-região, da regional ou da macro-região, e, além disso, identificará de que cidade é a responsabilidade de atender pacientes advindos de outras cidades, de acordo com a hierarquização e, assim, distribuir a verba pública de forma mais justa.

Um dos principais melhoramentos que o algoritmo proporcionará é a distribuição otimizada de pacientes entre as cidades do estado, principalmente para os procedimentos de baixa e média complexidade, pois se os pacientes que necessitarem de um atendimento nestas complexidades e não precisarem ir para os grandes centros, fará com que ocorra a diminuição das filas de espera nos grandes hospitais do estado. Através do procedimento de hierarquização, o algoritmo proporciona, ainda, um "giro" da economia local, pois se um paciente não se desloca de sua região para ser atendido, os gastos extras como, hotel para os seus parentes, alimentação, e outros, permanecerão na própria região de origem, beneficiando inclusive o comércio local, o que pode servir de estímulo à economia da cidade.

Atualmente, as divisões hierárquicas fazem com que as sedes das divisões sejam responsáveis pelas cidades relacionadas a ela; assim, uma cidade que não seja sede, terá no máximo quatro opções no processo de busca para solucionar o seu problema, as sedes das divisões em que ela se encontra e a capital do estado. O algoritmo apresentará opções adicionais à solução e, dessa forma, o fluxo de pacientes será mais homogêneo por todo o estado. É claro que para procedimentos médicos de alta complexidade, ainda será necessário recorrer aos grandes centros, principalmente a capital, porém essa procura por tais procedimentos, é um pequeno percentual do total.

Vale salientar sobre a importância da constante verificação dos dados com relação aos procedimentos realizados, fazendo com que o algoritmo apresente um desempenho cada vez mais satisfatório de acordo com progressivo ajuste dos mesmos.

Além disso, o algoritmo poderá melhorar ainda mais o seu desempenho se as divisões hierárquicas forem elaboradas de maneira mais otimizada do que a atual, utilizando técnicas adicionais da área da Pesquisa Operacional, para definir de forma ótima, as sedes das referidas divisões, baseando-se no problema de $p$-medianas [5] capacitado, o que fica como sugestão para trabalhos futuros. Abstract. This work presents an optimized solution for flow of patients seeking
for attendance in the "Public Health System" (PHS) in the state of Paraná, Brazil,
serving the situation where local facilities (the cities where the patients live) do not
have the medical procedure on need. The solution for the problem has to consider,
besides the required procedure, also, the step-by-step region hierarchy that, in a
crescent order is: city, micro-region, region and macro-region, from the place of the
user until his/her final destination; it is also to be considered the capacity in the
assigned place for the service. The problem consists in getting the optimal flow
definition for the PHS users from the original site until the nearest health center
with the requested service available, obeying the mentioned constraints, in order to
attend patients as fast as possible. This goal may be reached through a computati-
onal system, which includes a mathematical algorithm, that organizes and controls
the data of the Paraná State PHS in a suitable way; and as a consequence of that, it
will be possible as well to get a optimization for the financial resources the PHS has 
to assign to the municipalities. The developed tool, based on Operations Research techniques, is user friendly and performs with a great efficiency, presenting better results than the ones in use.

\section{Referências}

[1] R.H. Ballou, "Logística Empresarial", Ed. Atlas, São Paulo, SP, 1993.

[2] R.E. Campelo, N. Maculan, "Algoritmos e Heurísticas: Desenvolvimento e Avaliação de Performance", EDUFF, Niterói, RJ, 1994.

[3] N. Christofides, "Graph Theory: an Algorithmic Approach", Academic Press, London, 1975.

[4] T.H. Cormen, E.C. Leiserson, L.R. Rivest, "Introduction to Algorithms", MIT Press/McGraw-Hill, Cambridge, Massachusetts, 1990.

[5] M.C. Goldbarg, H.P.L. Luna, "Otimização Combinatória e Programação Linear - Modelos e Algoritmos", Editora Campus, RJ, 2000.

[6] L.L. Lapin, "Quantitative Methods for Business Decisions", Fort Worth, The Dryden Press, 1994.

[7] Ministério da Saúde, Portaria GM no. 1101, Brasília, DF, Brasil, 2002.

[8] C. Papadimitriou, K. Steiglitz, "Combinatorial Optimization-Algorithms and Complexity", Dover Publications, Mineola, New York, 1999. 